Few Body Systems manuscript No.

(will be inserted by the editor)

\title{
The strong interaction at the collider and cosmic-rays frontiers
}

\author{
David d'Enterria · Ralph Engel · Tanguy Pierog · Sergey \\ Ostapchenko · Klaus Werner
}

Received: date / Accepted: date

\begin{abstract}
First data on inclusive particle production measured in proton-proton collisions at the Large Hadron Collider (LHC) are compared to predictions of various hadron-interaction Monte Carlos (QGSJET, EPOS and SIBYLL) used commonly in high-energy cosmic-ray physics. While reasonable overall agreement is found for some of the models, none of them reproduces consistently the $\sqrt{s}$ evolution of all the measured observables. We discuss the implications of the new LHC data for the modeling of the non-perturbative and semihard parton dynamics in hadron-hadron and cosmic-rays interactions at the highest energies studied today.
\end{abstract}

Keywords Hadron-hadron collisions · Ultra-high-energy cosmic rays $\cdot$ LHC $\cdot$ QCD

\section{Introduction}

The highest energy hadronic interactions measured on Earth result from the collision of cosmic rays (CR) protons and nuclei accelerated in various astrophysical sources that propagate through the universe up to the so-called Greisen-Zatsepin-Kuzmin (GZK) cutoff around $10^{20} \mathrm{eV}[1,2]$ - with air nuclei in the upper atmosphere [3]. The determination of the primary energy and identity (mass) of such ultra-high-energy cosmic rays relies on the study of the cascade of secondary particles, called extensive air-showers (EAS), that they produce in the atmosphere [4], and its comparison to simulations that include the modeling of hadronic interactions at c.m. energies $\left(\sqrt{s_{G Z K}} \approx 400 \mathrm{TeV}\right)$ more than two orders of magnitude higher than those studied at particle colliders before the LHC. The dominant source of uncertainty in the interpretation of the highest-energy EAS data stems from our limitations to model particle production in strongly interacting systems. Indeed, even at asymptotically large energies the collision between two hadronic objects is sensitive to non-perturbative - hadronization, beam remnants, soft "peripheral" diffractive scatterings - or semi-hard - saturation of gluon densities, multi-parton interactions - dynamics that need still to be constrained directly from experimental data.

The first LHC data have extended by more than a factor of three the c.m. energies for which we have direct proton-proton measurements available to test and constrain the ingredients of the hadronic Monte Carlo (MC) codes used in CR physics. In this work we compare the predictions of several CR MCs with various inclusive observables measured at the LHC which are sensitive to non-perturbative and semihard QCD dynamics:

(i) total inelastic $p$ - $p$ cross sections $\sigma_{\text {inel }}$,

(ii) pseudorapidity density of charged particles at midrapidity $d N_{c h} /\left.d \eta\right|_{\eta=0}$,

(iii) event-by-event distribution of the charged particle multiplicity $P\left(N_{c h}\right)$,

Presented at the workshop "30 years of strong interactions", Spa, Belgium, 6-8 April 2011.

D. d'Enterria

CERN, PH Department, 1211 Geneva, Switzerland

E-mail: dde@cern.ch

R. Engel, T. Pierog

Karlsruhe Institut of Technology, Postfach 3640, 76021 Karlsruhe, Germany

S. Ostapchenko

NTNU, Inst. for Fysikk, 7491 Trondheim, Norway

K. Werner

SUBATECH, 4 rue Alfred Kastler, BP 20722, 44307 Nantes Cedex 3, France 
(iv) energy distribution of (very) forward particles $d N /\left.d E_{\gamma}\right|_{|\eta|>10.94}$ and $d E_{\text {had }} /\left.d \eta\right|_{|\eta|=3-5}$,

(v) average transverse momentum of the produced hadrons $\left\langle p_{\perp}\right\rangle$.

The implications of the data-theory comparisons for the improvement of the description of multiparticle production in the hadronic event generators and for the interpretation of CR results are discussed. The interested reader can find more details in [5,6].

\section{Hadronic collisions at multi-TeV energies}

The inclusive production of particles in high-energy hadronic collisions receives contributions from "soft" and "hard" interactions between the partonic constituents of the colliding hadrons. Soft (resp. hard) processes involve mainly $t$-channel partons of virtualities $q^{2}$ typically below (resp. above) a scale $Q_{0}^{2}$ of a few $\mathrm{GeV}^{2}$.

Soft scatterings give rise to production of hadrons with small transverse momenta $p_{\perp}$ and dominate hadronic collisions at low energies $(\sqrt{s} \lesssim 20 \mathrm{GeV})$. Although soft processes have a virtuality scale not far from $\Lambda_{Q C D} \approx 0.2 \mathrm{GeV}$ and thus cannot be treated within perturbative QCD (pQCD), predictions based on basic quantum field-theory principles - such as unitarity and analyticity of scattering amplitudes - as implemented in the Gribov's Reggeon Field Theory (RFT) [7], give a decent account of their cross sections in terms of the exchange of virtual quasiparticle states (Pomerons and Reggeons). At high energies the dominant soft contributions are from diffractive scatterings where one or both colliding hadrons survive the interaction and few particles are produced.

(Semi)hard parton-parton scatterings dominate the inelastic hadron production cross-sections for c.m. energies above a few hundreds of $\mathrm{GeV}$. Hard processes with large $\left|q^{2}\right| \gg \Lambda_{Q C D}^{2}$ can be treated within perturbative QCD in a collinear-factorized approach in terms of parton distribution functions (PDFs) in the hadron convoluted with the elementary parton-parton subprocess computable at a given order in the strong coupling constant $\alpha_{s}\left(q^{2}\right)$. The scattered quarks and gluons produce then collimated bunches of final-state hadrons (jets) in a branching process dominated by perturbative parton splittings described by the Dokshitzer-Gribov-Lipatov-Altarelli-Parisi (DGLAP) equations [8]9]10], and by non-perturbative hadronization (e.g. based on the Lund string model [11]) when the parton virtuality is below $\mathscr{O}(1 \mathrm{GeV})$. At increasingly larger c.m. energies, one needs to account for multi-parton scatterings and parton saturation effects. First, the cross section predicted by the (semi)hard processes exceeds the total inelastic $p$ - $p$ cross section for $p_{\perp}$ values of a few $\mathrm{GeV}$ indicating that multiple parton interactions (MPI) occur per collision. Second, for decreasing but still perturbative $p_{\perp}$ values, parton scatterings receive major contributions from the region of low fractional momenta $\left(x=p_{\text {parton }} / p_{\text {hadron }}\right)$, where the gluon distribution rises very fast. In this regime, around a "saturation scale" $Q_{\text {sat }}^{2}$ of a few $\mathrm{GeV}^{2}$, parton branching and fusion processes should start to compensate each other saturating the growth of the PDFs as $x \rightarrow 0$ [12].

The MC event generators of high-energy hadronic collisions used in CR physics - such as QGSJET01 and II [13] 14], SIBYLL [15, 16, 17] and EPOS [18] - have evolved starting up from the RFT approach, based on Pomeron degrees of freedom and thus naturally accounting for soft dynamics, generalized to include perturbative parton-parton processes via "cut (hard) Pomerons" diagrams. Multi-scattering phenomena (gluon saturation, MPI) are also implemented through various procedures [5].

\section{LHC data versus cosmic ray MCs}

Extensive air showers initiated by interactions of primary cosmic-ray particles with air nuclei in the atmosphere constitute multi-step cascade processes involving electromagnetic and hadronic processes. The electromagnetic part is well described theoretically, whereas the hadronic interactions are modeled as summarized in the previous Section. The most important EAS observables are the the depth in the atmosphere where the number of charged particles reaches its maximum $X_{\max }\left(\mathrm{g} / \mathrm{cm}^{2}\right)$, the number of particles at maximum $N_{\max }$, and the number of electromagnetic particles $\left(e^{ \pm}, \gamma\right)$ and muons $\left(\mu^{ \pm}\right)$at ground [19].

The relation between high-energy hadronic interactions and EAS observables has been studied numerically in detail in [20]. The depth of shower maximum $X_{\max }$ depends mainly on (i) the inelastic cross section $\left(\sigma_{\text {inel }}\right)$ of the primary particle with air nuclei, (ii) the corresponding energy fraction (inelasticity) transferred to secondary particles but the most energetic "leading" one emitted at very forward rapidities, relative to the primary particle, and (iii) the multiplicity $\left(N_{c h}\right)$ of the primary and subsequent very high-energy interactions, which defines how the energy is distributed to secondary particles and corresponding sub-showers (and results in a given $N_{\max }$ ). The rest of EAS properties at ground are closely related to $X_{\max }$ and $N_{\max }$. We discuss below how the LHC measurements constrain the collision-energy evolution of quantities such as $\sigma_{\text {inel }}, N_{c h}$, or the inelasticity. 
3.1 Inelastic $p-p$ cross section

A fundamental quantity of all CR models is the total hadronic cross section $\sigma_{t o t}$ and its separation into elastic and inelastic (and, in particular, diffractive) components. The measurement of $\sigma_{e l}$ is accessible thanks to various forward proton detectors such as TOTEM [21] and ALFA (ATLAS) [22] in the LHC tunnel area. The ATLAS [23] and CMS [24] experiments have already reported a value $\sigma_{\text {inel }} \approx 70 \mathrm{mb}$ at $\sqrt{s}=7 \mathrm{TeV}$ which seems to favour the lowest of the two (inconsistent) values previously measured at Tevatron $(1.8 \mathrm{TeV})$ pointing to a slightly slower increase of the hadron production cross sections with c.m. energy as in EPOS and QGSJET01 (Fig. 1 left).
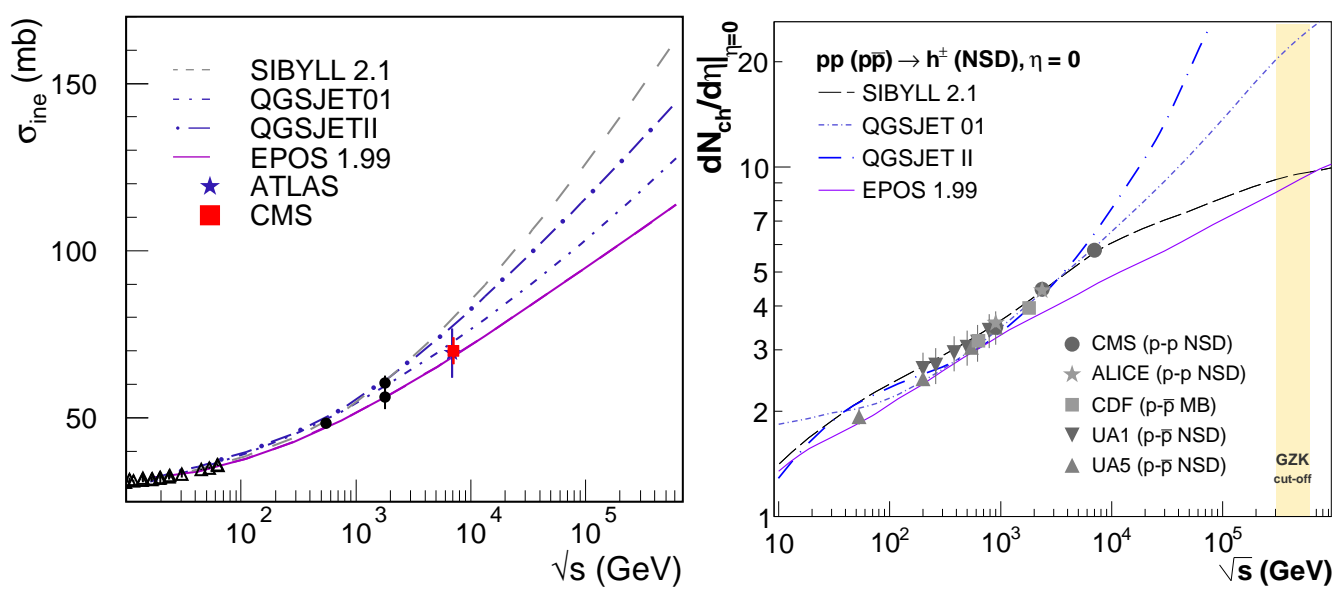

Fig. 1 Collision-energy dependence of the inelastic $p-p(p-\bar{p})$ cross section (left) and of the midrapidity charged hadron multiplicity density (right) including the latest LHC data and cosmic-ray MCs predictions.

\subsection{Charged particle multiplicity}

The charged hadron pseudorapidity density at LHC energies provides an important constraint for the modeling of the redistribution of energy in the first CR interactions in the atmosphere. The midrapidity measurements of $d N_{c h} /\left.d \eta\right|_{\eta=0} \approx 3.5,4.5,6$ at $\sqrt{s}=0.9,2.36,7 \mathrm{TeV}$ by ALICE [25], ATLAS [26] and CMS [27] indicate that the multiplicity changes smoothly in the lab energy range from $4 \times 10^{14}$ to $3 \times 10^{16} \mathrm{eV}$, being well reproduced (with the exception of EPOS) by the current interaction models used for EAS simulations (Fig. 1 right). In addition, first results on the heavy-ion $(\mathrm{Pb}-\mathrm{Pb})$ multiplicity and its centrality dependence at $\sqrt{s_{N N}}=2.76 \mathrm{TeV}$ [28] provide extra important cross-checks on the role of initial-state gluon saturation effects in collisions involving nuclei, such as those of $\mathrm{CR}$ with air.

\subsection{Multiplicity probability distributions}

The multiplicity distribution $P\left(N_{c h}\right)$, i.e. the probability to produce $N_{c h}$ charged hadrons in an event provides important differential constraints on the internal details of the hadronic interaction models. The low multiplicity part is mostly dominated by the contributions from diffraction (single-cut Pomeron exchanges), whereas the tail of the distribution gives information on the relative contribution of multiparton scatterings (multi-Pomeron exchanges). The experimental measurements (Fig. 2) at the three c.m. energies measured so far at the LHC, indicate that the high- $N_{c h}$ tail (left) is underestimated by EPOS and QGSJET01, whereas SIBYLL and QGSJETII get a bit closer, sometimes overestimating the data. In the low- $N_{c h}$ region around $P\left(N_{c h}\right) \sim 4$ (right panel), only EPOS globally reproduces the experimental results whereas the rest of the models overestimate the measurements up to $+30 \%$ for SIB YLL. The peak is even shifted towards lower multiplicity in the case of both QGSJET models. Thus, even if the average $p-p$ multiplicities are well reproduced by most models (Fig. 1 right), the details of their probability distributions are missed and indicate possible paths for improvement of the different model ingredients. 

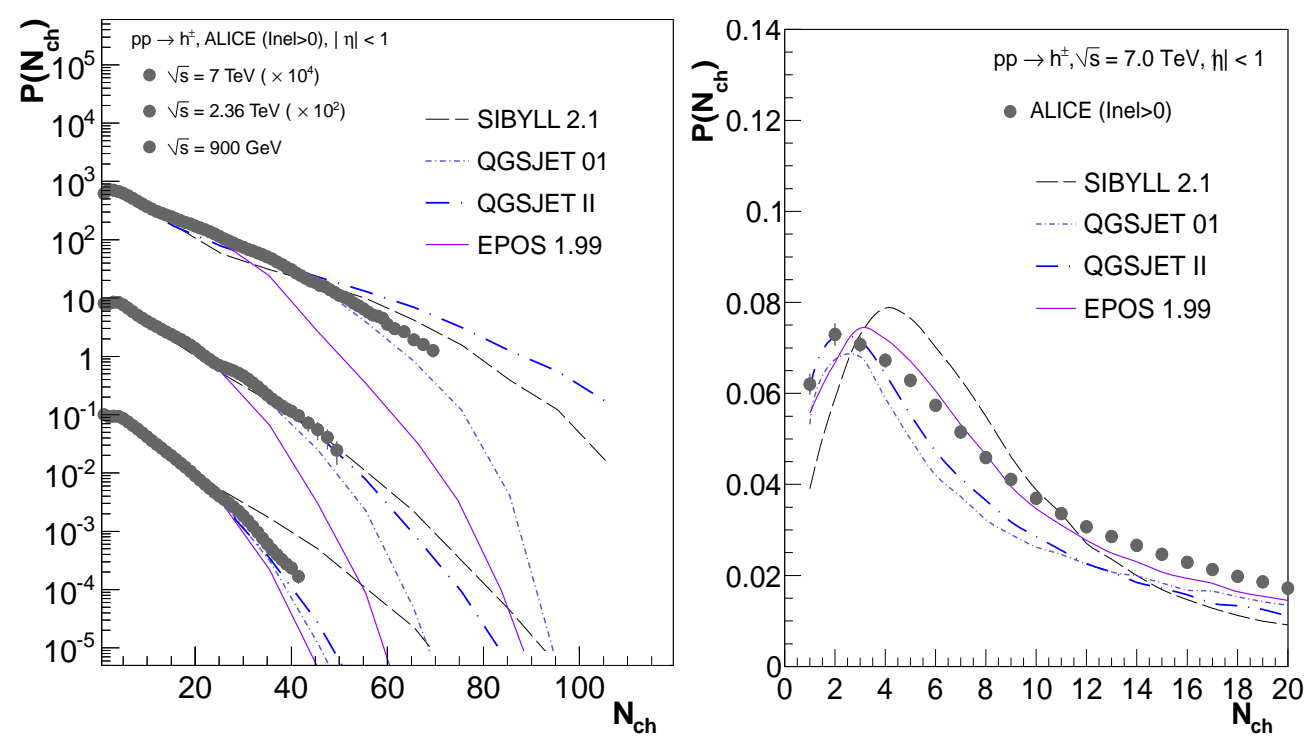

Fig. 2 Multiplicity distributions of charged hadrons, $P\left(N_{c h}\right)$, measured by ALICE in $p$ - $p$ events at $\sqrt{s}=0.9,2.36$ and 7 TeV [25] (left) compared to QGSJET01 and II, SIBYLL, and EPOS. The right plot shows a "zoom" in the low multiplicity range for $\sqrt{s}=7 \mathrm{TeV}$.

\subsection{Forward energy flow}

A key quantity for air shower development is the distribution of neutral energy emitted at very forward rapidities as it provides constraints on the production of leading hadrons (inelasticity) as well as on the transfer of energy from the hadronic core to the electromagnetic cascade (via $\pi^{0} \rightarrow \gamma \gamma$ decays). The recent LHCf measurement of the $\gamma$ spectrum for rapidities above $|\eta| \approx 8.8$ [29] is compared to model simulations in Fig. 3 (left) [6]. The simulations are in relatively good agreement with the data within the systematical uncertainty (not shown here) although for $E_{\gamma} \lesssim 1.5 \mathrm{TeV}$ the spectrum slope is harder in the data than in the predictions. Of similar importance is the measurement of the energy flow and particle spectra in the forward range $|\eta|=5-10$ [30]. This is an angular range that has been historically very difficult to access in collider experiments but that is partially covered by various detectors at the LHC such as TOTEM [21] and CASTOR (CMS) [31]. Preliminary results of the energy flow in the $|\eta|=3-5$ range indicate a good data-model agreement at $\sqrt{s}=0.9$ and $7 \mathrm{TeV}$ [32] (Fig. 3] right).
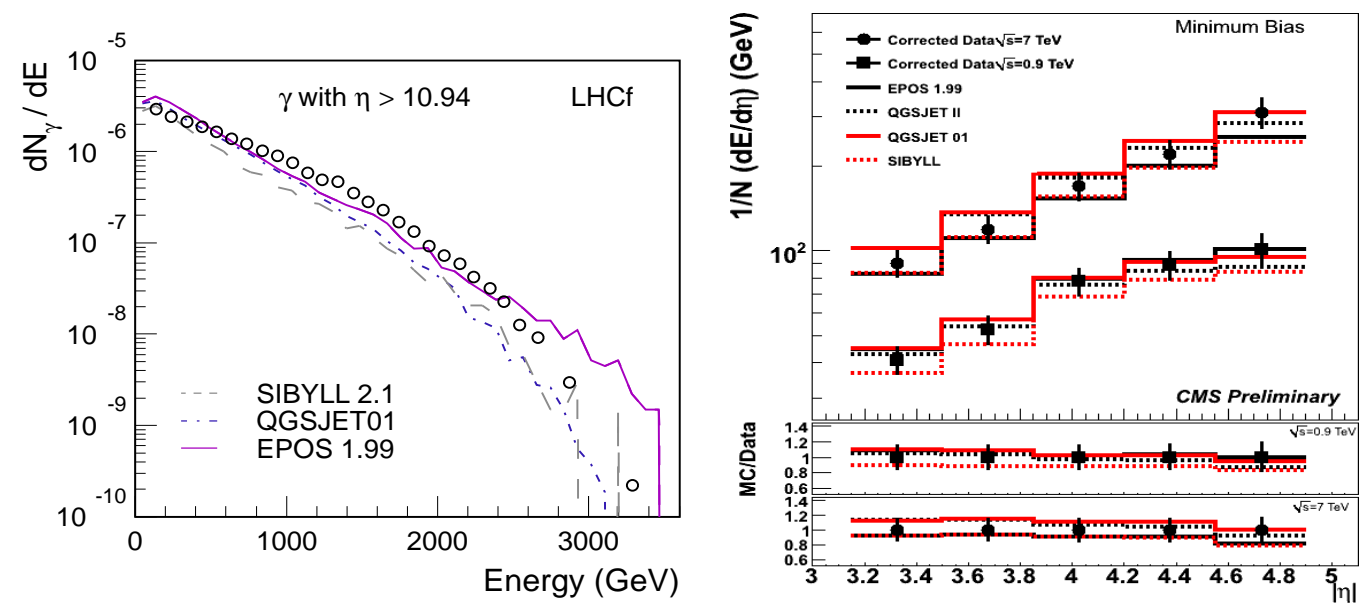

Fig. 3 Forward energy distributions in $p-p$ collisions at the LHC compared to CR Monte Carlos, for photons at $|\eta|>10.94$ measured by LHCf at $7 \mathrm{TeV}$ [29] (left) and for inclusive hadrons at $3<|\eta|<5$ measured by CMS at 0.9, $7 \mathrm{TeV}$ [32] (right). 
3.5 Transverse momenta of hadrons

Although the transverse momentum spectrum of the produced hadrons (or its average $p_{\perp}$ ) at the LHC does not have a direct impact on the interpretation of air shower data - the lateral distributions of particles at ground is rather defined by multiple Coulomb scattering and by the $p_{\perp}$ spectra of secondaries at much lower energies - such a measurement is of importance for checking the overall physics consistency of soft and hard interaction mechanisms implemented in the models. Indeed, at high energies the peak of the perturbative production comes from interactions between partons whose transverse momentum is around the saturation scale, $p_{\perp} \sim Q_{s a t}$, producing (mini)jets of a few $\mathrm{GeV}$ which fragment into hadrons. In models with saturation of parton densities, the mean transverse momentum of the produced hadrons is of the order of the saturation scale $Q_{s a t}$ in the high-energy limit.

In Fig. 4 (left) we show the energy evolution of the mean $p_{\perp}$ measured experimentally compared to the CR event generators and to the PYTHIA 8 MC [33]. All the RFT MCs but EPOS predict a very moderate increase of $\left\langle p_{\perp}\right\rangle$ with energy, reaching $\left\langle p_{\perp}\right\rangle \sim 0.6 \mathrm{GeV} / \mathrm{c}$ at GZK energies which is only $0.05 \mathrm{GeV} / \mathrm{c}$ above the current CMS result at $7 \mathrm{TeV}$, reflecting the moderate assumptions made on the saturation of the low- $x$ parton densities. EPOS predicts a significantly larger $\left\langle p_{\perp}\right\rangle_{G Z K} \approx 1 \mathrm{GeV} / \mathrm{c}$ due to the inclusion of final-state collective parton expansion effects. PYTHIA 8 - whose dynamics is dominated by (mini)jet production with a running $p_{\perp}$ cutoff that mimics parton saturation effects [5]-predicts a higher average $\left\langle p_{\perp}\right\rangle_{\text {GZK }} \approx 1.2 \mathrm{GeV} / \mathrm{c}$.
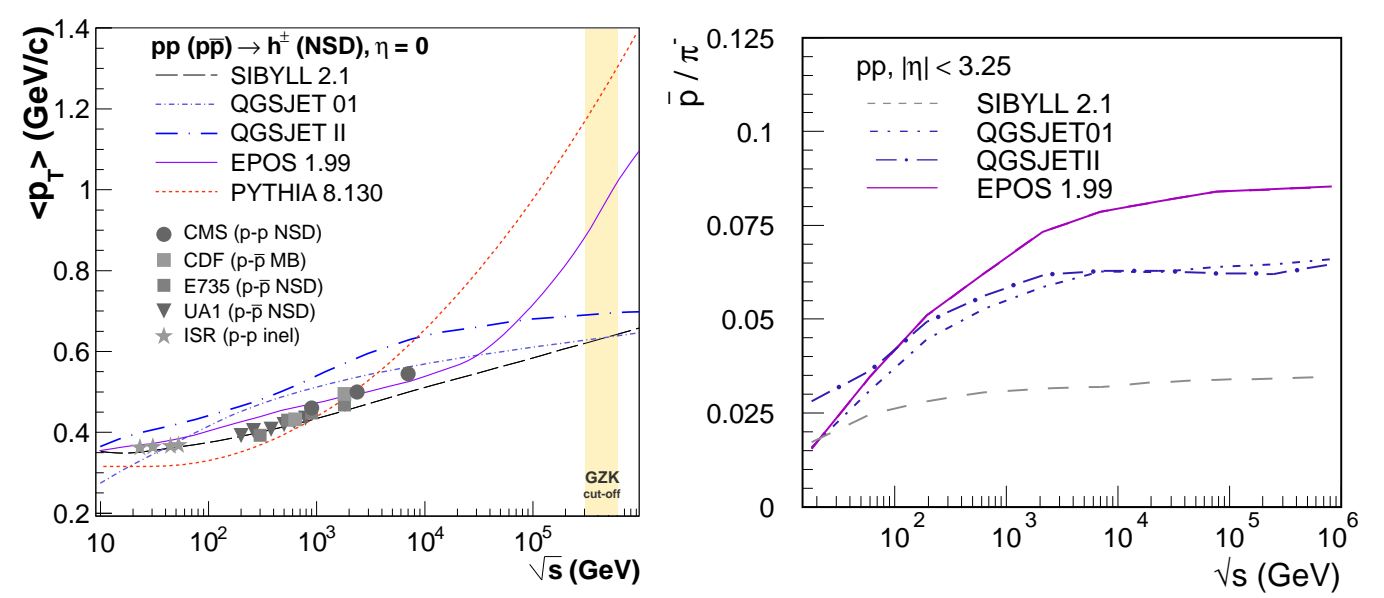

Fig. 4 Left: Average $p_{\perp}$ of charged particles at midrapidity in $p-p(p-\bar{p})$ collisions as a function of $\sqrt{s}$ compared to the predictions of CR event generators and PYTHIA 8. Right: Collision-energy dependence of the ratio of antiprotons to $\pi^{-}$yields at midrapidity predicted by various CR models.

The level of (dis)agreement between the inclusive hadron observables measured at the LHC discussed in [5] 6] and each one of the four CR hadronic MCs considered, is summarized in Table 1 The event generators give an overall decent description of all measurements but model improvements, particularly, those related to the treatment of inelastic diffraction and of the parton saturation mechanism, are desirable.

\begin{tabular}{lc|ccc|ccc|ccc|ccc}
\hline & Model & \multicolumn{3}{|c|}{ SIBYLL 2.1 } & \multicolumn{3}{|c|}{ QGSJET01 } & \multicolumn{3}{|c|}{ QGSJETII } & \multicolumn{3}{|c}{ EPOS 1.99 } \\
& $\sqrt{s}(\mathrm{TeV})$ & 0.9 & 2.36 & 7 & 0.9 & 2.36 & 7 & 0.9 & 2.36 & 7 & 0.9 & 2.36 & 7 \\
\hline$\sigma_{\text {inel }}$ & & $\checkmark$ & $\Uparrow$ & $\Uparrow$ & $\checkmark$ & $\checkmark$ & $\checkmark$ & $\checkmark$ & $\Uparrow$ & $\Uparrow$ & $\checkmark$ & $\checkmark$ & $\checkmark$ \\
$d N_{c h} /\left.d \eta\right|_{\eta=0}$ & & $\checkmark$ & $\checkmark$ & $\checkmark$ & $\checkmark$ & $\checkmark$ & $\checkmark$ & $\checkmark$ & $\checkmark$ & $\Uparrow$ & $\checkmark$ & $\Downarrow$ & $\Downarrow$ \\
$P\left(N_{c h}<5\right)$ & & $\Uparrow$ & $\Uparrow$ & $\Uparrow$ & $\Uparrow$ & $\Uparrow$ & $\Downarrow$ & $\Uparrow$ & $\Uparrow$ & $\Uparrow$ & $\checkmark$ & $\checkmark$ & $\checkmark$ \\
$P\left(N_{c h}>30\right)$ & & $\Uparrow$ & $\checkmark$ & $\Uparrow$ & $\checkmark$ & $\Downarrow$ & $\Downarrow$ & $\checkmark$ & $\checkmark$ & $\Uparrow$ & $\Downarrow$ & $\Downarrow$ & $\Downarrow$ \\
$\left\langle p_{\perp}\right\rangle$ & & $\checkmark$ & $\Downarrow$ & $\Downarrow$ & $\Uparrow$ & $\Uparrow$ & $\checkmark$ & $\Uparrow$ & $\Uparrow$ & $\Uparrow$ & $\checkmark$ & $\checkmark$ & $\checkmark$ \\
\hline
\end{tabular}

Table 1 Level of overall agreement between QGSJET01, QGSJETII, SIBYLL 2.1 and EPOS 1.99 with inclusive charged hadron results measured in collisions at $0.9,2.36$ and $7 \mathrm{TeV}$ : inelastic cross section $\sigma_{\text {inel }}$, pseudorapidity densities $d N_{c h} /\left.d \eta\right|_{\eta=0}$, multiplicity probabilities $P\left(N_{c h}\right)$ for low and high values of $N_{c h}$, and mean transverse momentum $\left\langle p_{\perp}\right\rangle$. A tick $(\checkmark)$ indicates a reasonable data-model agreement within experimental uncertainties, and $\Uparrow(\Downarrow)$ that the MC tends to over (under) estimate the measurements. 


\section{Conclusions}

Event generators used in high-energy cosmic ray (CR) physics include a description of hadronic interactions that partially depends on non- and semi-perturbative QCD dynamics that need to be calibrated with experimental data. The highest energy cosmic rays measured on Earth at $E_{l a b} \approx 10^{20} \mathrm{GeV}$ collide with the nuclei in the atmosphere at c.m. energies more than two orders of magnitude above those studied at particle colliders before the LHC. The most recent results from $p$ - $p$ collisions at the LHC (equivalent to lab energies around $E_{l a b}=3 \times 10^{16} \mathrm{eV}$ ) are of big help to constrain the details of multiparticle production in the Monte Carlos used to describe CR air showers. The measured characteristics of the bulk of hadron production at multi-TeV energies does not reveal serious deficiencies in any of the models. This gives a strong support to the interpretation of the results in the CR "knee" energy range $\left(E_{\text {lab }}=10^{15.5} \mathrm{eV}\right)$ in terms of conventional primary spectrum and nuclear mass composition and disfavours some proposed speculative ideas that the change of the CR spectral slope could be due to a sudden change in the hadronic interaction mechanism above $2 \mathrm{TeV}$ c.m. energy (see e.g. [34]).

Although the first LHC measurements support a conventional extrapolation of the known features of multiparticle production to the highest known energies, none of the models is in perfect agreement with all the hadronic observables measured at the LHC (see Table 1). In particular, extrapolations at the GZK-cutoff energies span a range of predictions - e.g. $d N_{c h} /\left.d \eta\right|_{\eta=0} \approx 10$ (EPOS, SIBYLL) - 50 (QGSJETII) for the particle densities and $\left\langle p_{\perp}\right\rangle \approx 0.6$ (SIBYLL, QGSJET01) - 1 (EPOS) GeV/c for the mean hadron transverse momentum - that justify the concurrent use of various MCs to gauge the uncertainties connected to hadronic interaction models in the interpretation of the cosmic ray data. New retuning of model parameters and reconsideration of model assumptions, e.g. for EPOS [35] and QGSJET [36], are currently underway.

Further improvement of our understanding of the strong interaction and of the properties of cosmic-rays at multi-TeV energies will be provided by the LHC with $p$ - $p$ data at the nominal c.m. energy of $\sqrt{s}=14 \mathrm{TeV}$ (corresponding to $\mathrm{CR}$ protons of $10^{17} \mathrm{eV}$ in the lab frame), as well as from the expected proton-nucleus ( $p$ $P b)$ runs at $\sqrt{S_{N N}}=8.8 \mathrm{TeV}$ [37], since the CR-induced air showers in the upper atmosphere are mostly from $(\mathrm{p}, \alpha, \mathrm{Fe})+(\mathrm{N}, \mathrm{O})$ collisions. Also, since baryon-induced subshowers lead to a higher number of muons at ground than meson-induced ones, the energy dependence of the baryon production rate (see e.g. Fig.44right), its relation to the centrality of the collision, and the momentum distribution of the baryons constitute also important quantities to be measured.

\section{References}

1. K. Greisen, Phys. Rev. Lett. 16 (1966) 748-750.

2. G. T. Zatsepin and V. A. Kuzmin, J. Exp. Theor. Phys. Lett. 4 (1966) 78

3. J. Blümer, R. Engel, and J. R. Hörandel, Prog. Part. Nucl. Phys. 63 (2009) 293-338

4. J. Knapp, D. Heck, S. J. Sciutto, M. T. Dova, and M. Risse, Astropart. Phys. 19 (2003) 77-99

5. D. d'Enterria, R. Engel, T. Pierog, S. Ostapchenko and K. Werner, Astropart. Phys. to appear; arXiv:1101.5596

6. T. Pierog, D. d'Enterria, R. Engel, S. Ostapchenko and K. Werner, Proceeds. ICRC'11.

7. V. N. Gribov, Sov. Phys. JETP 26 (1968) 414-422.

8. V. N. Gribov and L. N. Lipatov, Sov. J. Nucl. Phys. 15 (1972) $438-450$

9. G. Altarelli and G. Parisi, Nucl. Phys. B126 (1977) 298

10. Yu. L. Dokshitzer, Sov. Phys. JETP 46 (1977) 641

11. B. Andersson, G. Gustafson, G. Ingelman, and T. Sjostrand, Phys. Rept. 97 (1983) 31

12. L. V. Gribov, E. M. Levin, and M. G. Ryskin, Phys. Rept. 100 (1983) 1-150.

13. N. N. Kalmykov, S. S. Ostapchenko, and A. I. Pavlov, Nucl. Phys. Proc. Suppl. 52B (1997) 17-28

14. S. Ostapchenko, Nucl. Phys. Proc. Suppl. 151 (2006) 143-146

15. J. Engel, T. K. Gaisser, T. Stanev, and P. Lipari, Phys. Rev. D46 (1992) 5013-5025.

16. R. S. Fletcher, T. K. Gaisser, P. Lipari, and T. Stanev, Phys. Rev. D50 (1994) 5710-5731.

17. E.-J. Ahn, R. Engel, T. K. Gaisser, P. Lipari, and T. Stanev, Phys. Rev. D 80 (2009) 094003

18. K. Werner, F.-M. Liu, and T. Pierog, Phys. Rev. C74 (2006) 044902

19. A. Haungs, H. Rebel, and M. Roth, Rept. Prog. Phys. 66 (2003) 1145-1206.

20. R. Ulrich, R. Engel, and M. Unger, Phys. Rev. D 83 (2011) 054026 and arXiv:1010.4310 [hep-ph].

21. G. Anelli et al. (TOTEM Collab.), JINST 3 (2008) S08007.

22. S. Ask, arXiv:0706.0644 [hep-ex].

23. G. Aad et al. [ATLAS Collaboration], arXiv:1104.0326 [hep-ex].

24. M. Marone (CMS Collab.), Proceeds. DIS'11

25. K. Aamodt et al. (ALICE Collab.), Eur. Phys. J. C 68 (2010) 89; and Eur. Phys. J. C 68 (2010) 345

26. G. Aad et al. (ATLAS Collab.), arXiv:1012.5104 [hep-ex].

27. V. Khachatryan et al. (CMS Collab.), JHEP 02 (2010) 041; and Phys. Rev. Lett. 105 (2010) 022002

28. K. Aamodt et al. (ALICE Collab.), Phys. Rev. Lett. 106 (2011) 032301

29. O. Adriani et al. (LHCf Collab.) arXiv:1104.5294 [hep-ex].

30. D. d'Enterria, R. Engel, T. McCauley, and T. Pierog, Indian J. Phys. 84 (2010) 1837; arXiv:0806.0944

31. V. Andreev et al., Eur. Phys. J. C 67 (2010) 601 
32. A. Knutsson (CMS Collab.), Proceeds. DIS'11

33. T. Sjostrand, S. Mrenna, and P. Z. Skands, Comput. Phys. Commun. 178 (2008) 852-867; arXiv:0710.3820 [hep-ph].

34. R. Barceló, M. Masip, and I. Mastromatteo, JCAP 0906 (2009) 027; arXiv:0903.5247 [hep-ph].

35. T. Pierog, I. Karpenko, S. Porteboeuf, and K. Werner, arXiv:1011.3748 [astro-ph.HE]

36. S. Ostapchenko, Phys. Rev. D83 (2011) 014018; arXiv:1010.1869 [hep-ph].

37. C. A. Salgado et al., arXiv:1105.3919 [hep-ph]. 